\title{
Interfaz entre el plan de producción y el programa de producción en ambientes de ensamble
}

\author{
Interface between the production plan and the \\ master production schedule in assembly environments
}

Marcos Moya Navarro'

Magaly Sánchez Brenes² 


\section{Resumen}

En un entorno de producción existe una relación directa entre el mercado y los procesos de manufactura de los bienes. Sin embargo, cuando la producción está inmersa en un ambiente de ensamble, el procedimiento de la planificación y la programación se vuelve complejo, con el riesgo de que la empresa pierda ventaja competitiva en términos de incumplimiento de las fechas de entrega y elevados costos de planificación y control. La programación lineal constituye una herramienta apropiada para construir modelos de programación y control de la producción en entornos de manufactura complejos.

El objetivo de este trabajo es mostrar la interfaz entre el plan de producción y el programa de producción en ambientes de ensamble mediante el modelado por programación lineal. Para ello, se seleccionó un entorno que produce tres productos finales cuya estructura de producto está dispuesta en tres niveles con un máximo de ocho componentes. Los datos proporcionados por el plan de producción se desglosaron en diez órdenes, cada una indicando el tipo de producto, la cantidad por producir y su respectiva fecha de entrega. Los resultados obtenidos en el programa de producción establecieron la recepción de pedidos planificados y el programa de componentes totales requeridos en cada periodo de producción, para minimizar los costos totales de emisión de órdenes de producción e inventario.

\section{Palabras clave}

Ingeniería, industria, investigación de operaciones, ciencias de la computación, programación matemática.

\begin{abstract}
In a production environment there is a direct relationship between the market and the manufacturing process of goods. When production is immersed in an assembly environment, the process of production planning and scheduling becomes complex, and the enterprises have the risk of losing competitive advantages in terms of not meeting delivery dates and production high costs. Linear programming has become an appropriate tool for production planning and scheduling in complex manufacturing environments.

The aim of this paper is to show the interface between both the production process planning and the scheduling process in assembly environments by means of linear programming. The manufacturing process selected is capable of producing three products with a product structure arranged on three levels with a maximum of eight components each. Data provided by the aggregate production planning was grouped into ten production orders with information of product type, quantity to be produced and their respective delivery date. Final results were orders placement and components required in order to minimizing the cost of issuing orders and inventory costs.
\end{abstract}

\section{Key words}

Engineering, industry, operations research, computer sciences, mathematical programming. 


\section{Introducción}

En un entorno de producción hay una relación directa entre los clientes externos (mercado) y los procesos de producción. La demanda de los productos hace que estos sean fabricados y luego enviados al mercado para satisfacer tal demanda (Sipper et al., 1998). El establecimiento de los pronósticos de la demanda de los productos que generan los mercados inicia el proceso de planeamiento de la producción, el cual es un indicador de la producción total que deben presentar las compañías.

El problema que se genera en este proceso de planificación es que, además de los múltiples productos por fabricar, muchos de ellos están compuestos por ensambles y sub ensambles de partes. Algunas de las partes que componen los sub ensambles se fabrican en esos mismos entornos de producción, mientras que otras deben comprarse a proveedores externos. En consecuencia, se requiere elaborar un Programa Maestro de Producción (PMP), el cual separa el plan agregado de producción' en productos individuales.

El planeamiento agregado de la producción mediante programación matemática busca determinar el tamaño óptimo del lote de producción ${ }^{2}$ para cada uno de los productos o familias de productos que se van a fabricar, durante un horizonte de planeación especificado. Hanssmann y Hess (1960) fueron los primeros en formular un plan de producción mediante programación lineal (Moya, 20 I I).

La programación maestra de la producción en un entorno de ensamble busca determinar la cantidad total de materiales (partes) que se necesitan para cumplir con la entrega de los diferentes pedidos de productos cumpliendo con las fechas establecidas, siendo estas entregas justo a tiempo ${ }^{3}$. Esto significa que se penalizan las entregas antes o después de las fechas pactadas. El programa de producción se alimenta de los tamaños óptimos de producción recomendados en el plan agregado. La figura I

I Se denomina plan agregado de producción a aquel que incorpora el planeamiento de la producción de múltiples productos en el mismo plan.

2 Se considera un tamaño óptimo del lote de producción aquel que genera el menor costo total del plan de producción.

3 Entregas justo a tiempo significa entregar las cantidades requeridas de productos en las fechas pactadas; por lo tanto, se penaliza la entrega temprana y la entrega tardía. resume el orden en que debe realizarse el proceso de planeamiento y programación de la producción en ambientes de ensamble.

El objetivo de este trabajo es mostrar la interfaz entre el plan de producción y el programa de producción cuando los productos a producir se componen de sub ensambles y componentes individuales. El proceso de planeamiento y programación de la producción en ambientes de ensamble se modelará mediante el uso de la programación matemática con programación lineal, de tal manera que el programa de emisión de órdenes de producción (programación maestra de la producción) tome como punto de partida el conjunto de resultados proporcionados por el plan agregado de producción.

En consecuencia, el modelo de programación maestra de la producción permitirá determinar si el programa de emisión de órdenes propuesto es viable, en términos del cumplimiento de las fechas de entrega pactadas y además proporcionará el plan de componentes totales requeridos en cada periodo para cumplir con las demandas establecidas en el plan de producción para todos los productos.

\section{Ambiente de producción propuesto}

Se propone un ambiente de producción que consta de tres productos finales, cada uno de los cuales se debe producir en las cantidades que establece el plan agregado de producción. Cada producto final se compone de varios sub ensambles, según lo indica la estructura de producto correspondiente, dispuesto en tres niveles.

El resultado final obtenido consistirá del plan de componentes totales de cada tipo que se requerirán en cada periodo del plan de producción, de tal manera que se minimicen los costos totales de inventario y de penalización por entregas no justo a tiempo.

El ambiente propuesto consta de un centro de manufactura que produce tres tipos de productos especiales. El Departamento de Planificación y Control de la Producción tiene en este momento la responsabilidad de realizar el plan agregado de la producción para los próximos tres periodos. 


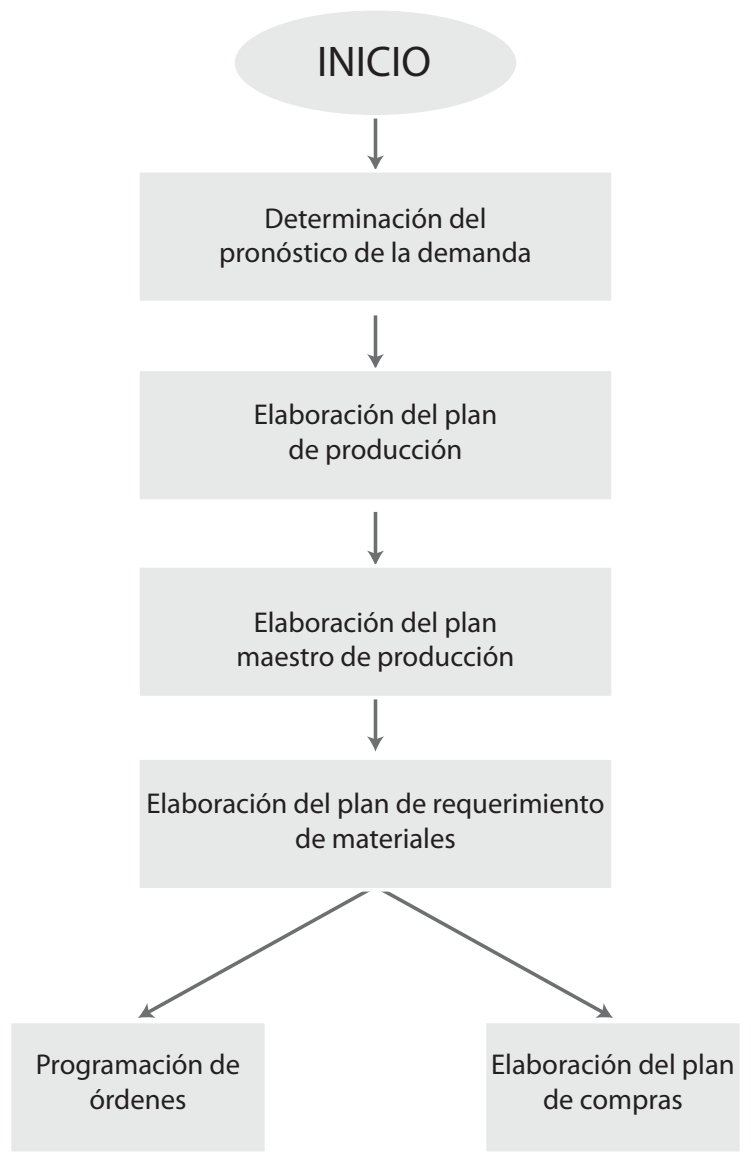

Figura I. Secuencia de pasos para realizar el programa maestro de producción en ambientes de ensamble

Cuadro I. Demanda pronosticada para cada producto y en cada periodo

\begin{tabular}{|c|c|c|c|c|}
\hline & \multicolumn{3}{|c|}{ Demanda pronosticada } & Horas \\
\hline Periodo & Producto I & Producto 2 & Producto 3 & Disponibles \\
\hline 1 & 350 & 300 & & 560 \\
\hline 2 & 650 & 600 & 100 & 560 \\
\hline 3 & 350 & 200 & 300 & 560 \\
\hline
\end{tabular}

Cuadro 2. Inventario inicial, tiempos de procesamiento y costos de emisión de órdenes y mantenimiento del inventario

\begin{tabular}{|c|c|c|c|}
\hline & Producto I & Producto 2 & Producto 3 \\
\hline Inventario inicial (unidades) & 50 & 25 & 30 \\
\hline Tiempo de procesamiento (horas) & 0.5 & 0.6 & 0.5 \\
\hline $\begin{array}{c}\text { Costo de emitir una orden de producción } \\
\text { Costo de mantenimiento de inventario (\$/Unidad- } \\
\text { Periodo) }\end{array}$ & 500 & 400 & 500 \\
\hline
\end{tabular}


Se dispone de información relativa a pronósticos de la demanda para cada producto en cada periodo del plan, las cantidades de inventario inicial de cada producto, los tiempos de procesamiento unitarios para cada producto, así como los costos de emisión de órdenes de producción y mantenimiento del inventario. Los cuadros 1 y 2 resumen la información.

Los productos por fabricar están compuestos de una serie de componentes que deben ser ensamblados a partir de un máximo de ocho. Las figuras 2, 3 y 4 muestran la estructura de producto para cada uno de los productos por fabricar.

La figura 2 indica que para fabricar el Producto I se requieren dos subensambles \#I, tres subensambles \#2, un subensamble \#3 y dos subensambles \#4. A su vez, para fabricar el subensamble \# I se requieren dos componentes \#5 y tres componentes \#6. Para fabricar el subensamble \#2 se requieren dos componentes \#6 y un componente \#7. Para manufacturar el subensamble \#3 se requieren un componente \#5, tres componentes \#6, dos componentes \#7 y un componente \#8. Finalmente, para producir el subensamble \#4 se requieren tres componentes \#6, dos componentes \#7 y tres componentes \#8. De manera similar, se interpreta la estructura de componentes que se muestran en la figuras 3 y 4.

El cuadro 3 muestra el inventario inicial de componentes, así como los costos respectivos de mantenimiento de inventario por unidad.

\section{Modelado del plan agregado de producción}

Con base en los datos presentados en los cuadros I y 2, se formuló el modelo de planificación agregada de la producción (Sipper et al., 1998) que se presenta en la figura 5, de tal manera que se minimicen los costos de emisión de órdenes de producción y los costos de mantener el inventario de los productos fabricados. El modelo propuesto en la figura 5 se resolvió en el programa de computadora OR Brainware Decision Tools desarrollado por Marcos Moya Navarro, Ph.D. (ver página web www.orbrainware.com)

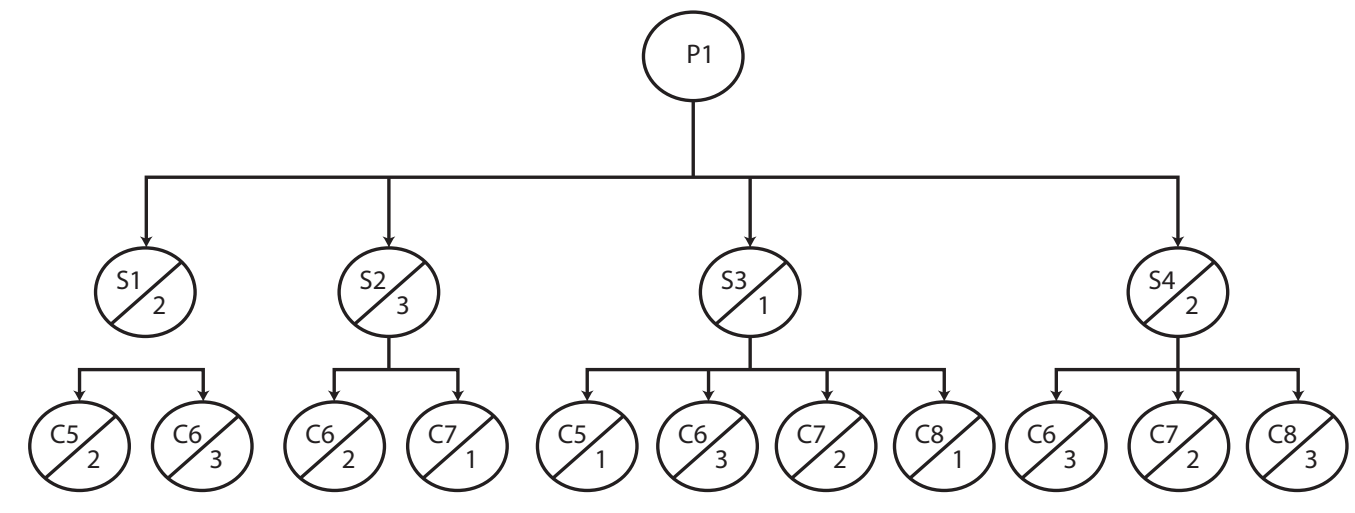

Figura 2. Estructura de componentes para el producto \# I.

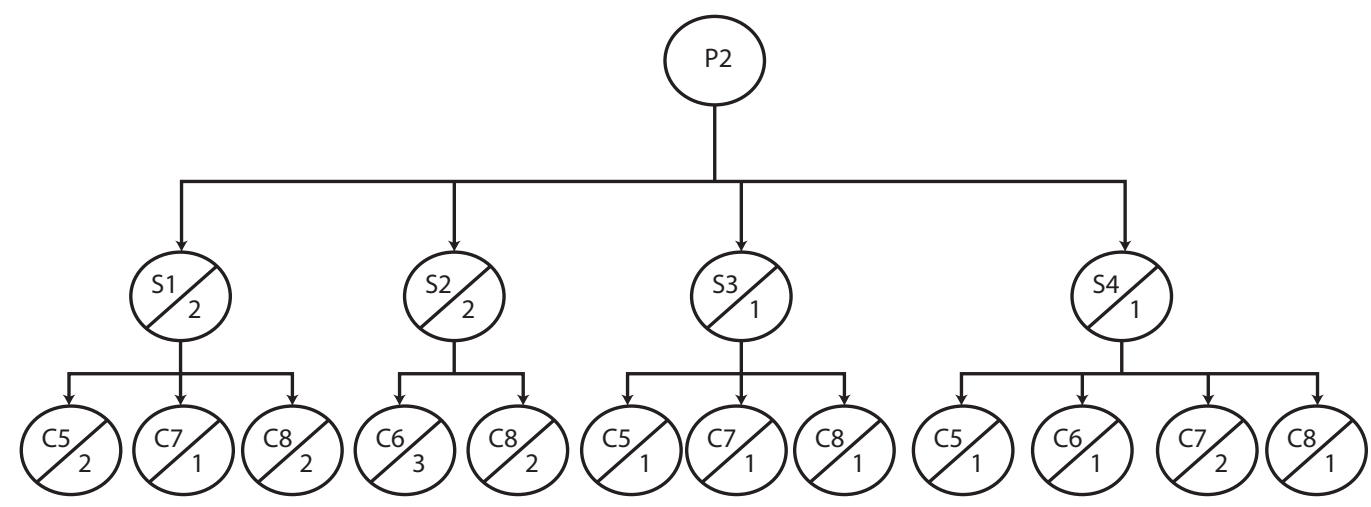

Figura 3: Estructura de componentes para el producto \#2 
Cuadro 3. Inventario inicial de componentes y costo de mantenimiento de este

\begin{tabular}{|c|c|c|c|c|c|c|c|c|}
\hline Componentes & S1 & S2 & S3 & S4 & C5 & C6 & C7 & C8 \\
\hline Inventario inicial & 300 & 500 & 150 & 90 & 250 & 100 & 75 & 200 \\
\hline Costo mantenimiento del inventario & 1.0 & 1.50 & 1.50 & 0.80 & 0.75 & 1.25 & 1.0 & 0.90 \\
\hline
\end{tabular}

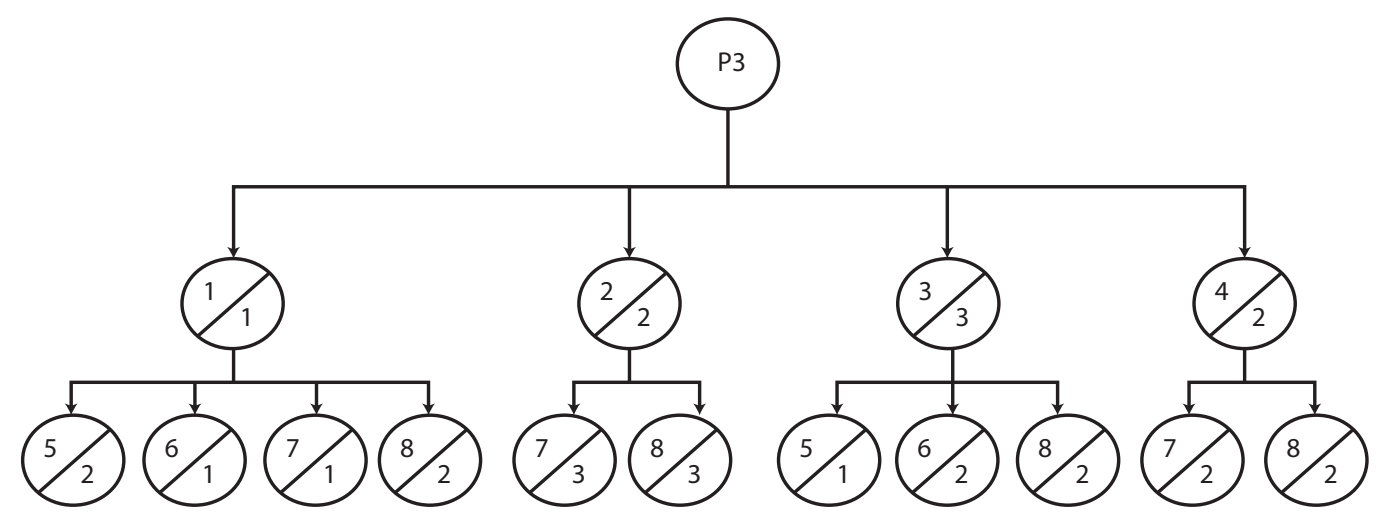

Figura 4. Estructura de componentes para el producto 3.

Min $\mathrm{X}_{\mathrm{o}}=600 \mathrm{Y}_{11}+600 \mathrm{Y}_{12}+600 \mathrm{Y}_{13}+400 \mathrm{Y}_{21}+400 \mathrm{Y}_{22}+400 \mathrm{Y}_{23}+500 \mathrm{Y}_{31}+$ $500 \mathrm{Y}_{32}+500 \mathrm{Y}_{33}+5 \mathrm{I}_{11}+5 \mathrm{I}_{12}+5 \mathrm{I}_{13}+4 \mathrm{I}_{21}+4 \mathrm{I}_{22}+4 \mathrm{I}_{23}+6 \mathrm{I}_{31}$ $+6 \mathrm{I}_{32}+6 \mathrm{I}_{33}+0 \mathrm{Q}_{11}+0 \mathrm{Q}_{12}+0 \mathrm{Q}_{13}+0 \mathrm{Q}_{21}+0 \mathrm{Q}_{22}+0 \mathrm{Q}_{23}$ $+0 \mathrm{Q}_{31}+0 \mathrm{Q}_{32}+0 \mathrm{Q}_{33}$

S.A.

$-1 \mathrm{I}_{11}+1 \mathrm{Q}_{11}=400$

$1 \mathrm{I}_{11}-1 \mathrm{I}_{12}+1 \mathrm{Q}_{12}=650$

$1 \mathrm{I}_{12}-1 \mathrm{I}_{13}+1 \mathrm{Q}_{13}=350$

$-1 \mathrm{I}_{21}-1 \mathrm{Q}_{21}=275$

$1 \mathrm{I}_{21}-1 \mathrm{I}_{22}+1 \mathrm{Q}_{22}=600$

$1 \mathrm{I}_{22}-1 \mathrm{I}_{23}+1 \mathrm{Q}_{23}=200$

$-1 \mathrm{I}_{31}+1 \mathrm{Q}_{31}=0$

$1 \mathrm{I}_{31}-1 \mathrm{I}_{32}+1 \mathrm{Q}_{32}=100$

$1 \mathrm{I}_{32}-1 \mathrm{I}_{33}+1 \mathrm{Q}_{33}=300$

$.5 \mathrm{Q}_{11}+.6 \mathrm{Q}_{21}+.5 \mathrm{Q}_{31} \leq 560$

$.5 \mathrm{Q}_{12}+.6 \mathrm{Q}_{22}+.5 \mathrm{Q}_{32} \leq 560$

$.5 \mathrm{Q}_{13}+.6 \mathrm{Q}_{23}+.5 \mathrm{Q}_{33} \leq 560$

$-1450 \mathrm{Y}_{11}+1 \mathrm{Q}_{11} \leq 0$

$-1000 \mathrm{Y}_{12}+1 \mathrm{Q}_{12} \leq 0$

$-350 \mathrm{Y}_{13}+1 \mathrm{Q}_{13} \leq 0$

$-1100 \mathrm{Y}_{21}+1 \mathrm{Q}_{21} \leq 0$

$-800 \mathrm{Y}_{22}+1 \mathrm{Q}_{22} \leq 0$

$-200 \mathrm{Y}_{23}+1 \mathrm{Q}_{23} \leq 0$

$-400 \mathrm{Y}_{31}+1 \mathrm{Q}_{31} \leq 0$

$-400 \mathrm{Y}_{32}+1 \mathrm{Q}_{32} \leq 0$

$-600 \mathrm{Y}_{33}+1 \mathrm{Q}_{33} \leq 0$

$\mathrm{Q}_{\mathrm{it}} \geq \mathrm{O} ; \quad \mathrm{I}_{\mathrm{it}} \geq \mathrm{O} ; \quad \mathrm{Y}_{\mathrm{it}}=0,1 ; \quad \forall \mathrm{i}$ y $\forall \mathrm{t}$

Figura 5. Formulación del Plan Agregado de Producción 
Cuadro 4. Plan de producción que minimiza los costos de emisión de órdenes y el mantenimiento del inventario

\begin{tabular}{|c|c|c|c|}
\hline \multirow{2}{*}{ Periodo } & \multicolumn{3}{|c|}{ Cantidades por producir } \\
\cline { 2 - 4 } & Producto I & Producto 2 & Producto 3 \\
\hline 1 & 300 & 567 & 0 \\
\hline 2 & 650 & 308 & 100 \\
\hline 3 & 350 & 200 & 300 \\
\hline
\end{tabular}

Cuadro 5. Emisión de órdenes con base en el plan de producción

\begin{tabular}{|c|c|c|c|c|c|c|c|c|c|c|}
\hline Orden & 1 & 2 & 3 & 4 & 5 & 6 & 7 & 8 & 9 & 10 \\
\hline Cantidad & 200 & 100 & 567 & 400 & 308 & 100 & 250 & 200 & 300 & 350 \\
\hline Producto & 1 & 1 & 2 & 1 & 2 & 3 & 1 & 2 & 3 & 1 \\
\hline Fecha de entrega & 1 & 1 & 1 & 2 & 2 & 2 & 2 & 3 & 3 & 3 \\
\hline Horas requeridas & 100 & 50 & 340,2 & 200 & 184,8 & 50 & 125 & 120 & 150 & 175 \\
\hline
\end{tabular}

Cuadro 6. Cantidad de componentes necesarios por orden emitida

\begin{tabular}{|c|c|c|c|c|c|c|c|c|c|c|}
\hline \multirow{2}{*}{ Componente } & \multicolumn{10}{|c|}{ Emisión de órdenes } \\
\cline { 2 - 12 } & $\mathbf{1}$ & $\mathbf{2}$ & $\mathbf{3}$ & $\mathbf{4}$ & $\mathbf{5}$ & $\mathbf{6}$ & $\mathbf{7}$ & $\mathbf{8}$ & $\mathbf{9}$ & $\mathbf{1 0}$ \\
\hline SI & 400 & 200 & 1134 & 800 & 616 & 100 & 500 & 400 & 300 & 700 \\
\hline S2 & 600 & 300 & 1134 & 1200 & 616 & 200 & 750 & 400 & 600 & 1050 \\
\hline S3 & 200 & 100 & 567 & 400 & 308 & 300 & 250 & 200 & 900 & 350 \\
\hline S4 & 400 & 200 & 567 & 800 & 308 & 200 & 500 & 200 & 600 & 700 \\
\hline C5 & 1000 & 500 & 3402 & 2000 & 1848 & 500 & 1250 & 1200 & 1500 & 1750 \\
\hline C6 & 4200 & 2100 & 3969 & 8400 & 2156 & 700 & 5250 & 1400 & 2100 & 7350 \\
\hline C7 & 1800 & 900 & 2835 & 3600 & 1540 & 1100 & 2250 & 1000 & 3300 & 3150 \\
\hline C8 & 1400 & 700 & 5670 & 2800 & 3080 & 1800 & 1750 & 2000 & 5400 & 2450 \\
\hline
\end{tabular}

En el modelo presentado en la figura 5, las primeras nueve restricciones determinan el inventario final de todos los productos en todos los periodos en función de las cantidades por producir y las demandas pronosticadas. Las siguientes tres restricciones garantizan que las cantidades óptimas de productos por producir no excedan la capacidad de los recursos disponibles en cada periodo, mientras que el último grupo de nueve restricciones garantiza que la producción total nunca excederá la demanda acumulada durante el horizonte de planeación. La figura 6 muestra el informe de respuestas obtenido por el programa OR Brainware Decision Tools.

El cuadro 4 resume los resultados presentados en la figura 6.

\section{Interfaz con el programa de} producción y el plan de materiales

Con base en la información suministrada por el plan agregado de producción (cuadro 4), el Departamento de Programación de la Producción emitió diez órdenes de producción para los productos individuales (desagregados), indicando el tipo de producto por fabricar, la cantidad de producción y la respectiva fecha de entrega.

El cuadro 5 muestra que para las 300 unidades del producto $\mathrm{PI}$, que según indican los resultados del plan de producción deben producirse en el periodo I, se emitieron dos órdenes de producción, la \# I por 200 unidades y la \#2 por 100 unidades, ambas con fecha de entrega en el periodo I. 


\begin{tabular}{|c|c|c|c|c|c|}
\hline 4 & A & C & D & $E$ & $\mathrm{~F}$ \\
\hline 1 & \\
\hline 2 & \multicolumn{3}{|c|}{ Hoja de Cálculo: [OR Brainware Decision Tools.xIsm]Hoja1 } & & \\
\hline 3 & \multicolumn{5}{|c|}{ Reporte Creado: 29/10/2012 10:32:41 a.m. } \\
\hline 4 & & & & & \\
\hline 5 & \multicolumn{5}{|l|}{ Objective Cell (Max) } \\
\hline 6 & Cell & Name & Original Value & Final Value & \\
\hline 7 & $\$ E \$ 509$ & Y11 & 0 & 4523,517245 & \\
\hline 8 & & & & & \\
\hline 9 & \multicolumn{5}{|l|}{ Variable Cells } \\
\hline 10 & Cell & Name & Original Value & Final Value & Integer \\
\hline 11 & \$E $\$ 5$ & Y11 & 0 & 0,275862069 & Contin \\
\hline 12 & $\$ F \$ 5$ & $\mathrm{Y} 12$ & 0 & 0,65 & Contin \\
\hline 13 & $\$ G \$ 5$ & Y13 & 0 & & Binary \\
\hline 14 & $\$ \mathrm{H} \$ 5$ & Y21 & 0 & & Binary \\
\hline 15 & $\$ 1 \$ 5$ & Y22 & 0 & & Binary \\
\hline 16 & $\$ J \$ 5$ & Y23 & 0 & & Binary \\
\hline 17 & $\$ K \$ 5$ & Y31 & 0 & & Binary \\
\hline 18 & $\$ L \$ 5$ & Y32 & 0 & & Binary \\
\hline 19 & $\$ M \$ 5$ & Y33 & 0 & & Binary \\
\hline 20 & $\$ N \$ 5$ & $\mid 11$ & 0 & & Contin \\
\hline 21 & $\$ O \$ 5$ & 112 & 0 & & Contin \\
\hline 22 & $\$ P \$ 5$ & 113 & 0 & & Contin \\
\hline 23 & $\$ Q \$ 5$ & 121 & 0 & 292 & Contin \\
\hline 24 & $\$ R \$ 5$ & 122 & 0 & & Contin \\
\hline 25 & $\$ S \$ 5$ & 123 & 0 & & Contin \\
\hline 26 & $\$ T \$ 5$ & 131 & 0 & & Contin \\
\hline 27 & \$U\$5 & 132 & 0 & & Contin \\
\hline 28 & $\$ V \$ 5$ & 133 & 0 & & Contin \\
\hline 29 & $\$ W \$ 5$ & Q11 & 0 & 400 & Integer \\
\hline 30 & $\$ \times \$ 5$ & Q12 & 0 & 650 & Integer \\
\hline 31 & $\$ Y \$ 5$ & Q13 & 0 & 350 & Integer \\
\hline 32 & $\$ Z \$ 5$ & Q21 & 0 & 567 & Integer \\
\hline 33 & \$AA\$5 & Q22 & 0 & 308 & Integer \\
\hline 34 & $\$ A B \$ 5$ & Q23 & 0 & 200 & Integer \\
\hline 35 & \$AC\$5 & Q31 & 0 & & Integer \\
\hline 36 & \$AD\$5 & Q32 & 0 & 100 & Integer \\
\hline 37 & $\$ A E \$ 5$ & Q33 & 0 & 300 & Integer \\
\hline
\end{tabular}

Figura 6. Solución óptima del modelo formulado en la figura 3. 
$\operatorname{Min} \mathrm{X}_{\mathrm{o}}=1 \mathrm{I}_{11}+1 \mathrm{I}_{12}+1 \mathrm{I}_{13}+1.5 \mathrm{I}_{21}+1.5 \mathrm{I}_{22}+1.5 \mathrm{I}_{23}+1.5 \mathrm{I}_{31}+1.5 \mathrm{I}_{32}+1.5 \mathrm{I}_{33}+0.8 \mathrm{I}_{41}+0.8 \mathrm{I}_{42}+0.8 \mathrm{I}_{43}+0.75 \mathrm{I}_{51}+0.75 \mathrm{I}_{52}+0.75 \mathrm{I}_{53}+1.25 \mathrm{I}_{61}+1.5 \mathrm{I}_{62}+$ $1.25 \mathrm{I}_{63}+1 \mathrm{I}_{71}+1 \mathrm{I}_{72}+1 \mathrm{I}_{73}+0.9 \mathrm{I}_{31}+0.9 \mathrm{I}_{32}+0.9 \mathrm{I}_{33}+0 \mathrm{Y}_{11}+2 \mathrm{Y}_{12}+4 \mathrm{Y}_{13}+0 \mathrm{Y}_{21}+1 \mathrm{Y}_{22}+2 \mathrm{Y}_{23}+0 \mathrm{Y}_{31}+3 \mathrm{Y}_{32}+$ $6 \mathrm{Y}_{33}+3 \mathrm{Y}_{41}+0 \mathrm{Y}_{42}+4 \mathrm{Y}_{43}+2 \mathrm{Y}_{51}+0 \mathrm{Y}_{52}+3 \mathrm{Y}_{53}+1.5 \mathrm{Y}_{61}+0 \mathrm{Y}_{62}+2 \mathrm{Y}_{63}+.9 \mathrm{Y}_{71}+0 \mathrm{Y}_{72}+1.2 \mathrm{Y}_{73}+2 \mathrm{Y}_{31}+4 \mathrm{Y}_{32}+0 \mathrm{Y}_{33}+3.5 \mathrm{Y}_{91}+$ $7 \mathrm{Y}_{92}+0 \mathrm{Y}_{93}+2.5 \mathrm{Y}_{101}+5 \mathrm{Y}_{102}+0 \mathrm{Y}_{103}+0 \mathrm{Q}_{11}+0 \mathrm{Q}_{12}+0 \mathrm{Q}_{13}+0 \mathrm{Q}_{21}+0 \mathrm{Q}_{22}+0 \mathrm{Q}_{23}+0 \mathrm{Q}_{31}+0 \mathrm{Q}_{32}+0 \mathrm{Q}_{33}+0 \mathrm{Q}_{41}+0 \mathrm{Q}_{42}+$ $0 \mathrm{Q}_{43}+0 \mathrm{Q}_{51}+0 \mathrm{Q}_{52}+0 \mathrm{Q}_{53}+0 \mathrm{Q}_{61}+0 \mathrm{Q}_{62}+0 \mathrm{Q}_{63}+0 \mathrm{Q}_{71}+0 \mathrm{Q}_{72}+0 \mathrm{Q}_{73}+0 \mathrm{Q}_{81}+0 \mathrm{Q}_{82}+0 \mathrm{Q}_{83}$

S.A.

$-1 \mathrm{I}_{11}-400 \mathrm{Y}_{11}-200 \mathrm{Y}_{21}-1134 \mathrm{Y}_{31}-800 \mathrm{Y}_{41}-616 \mathrm{Y}_{51}-100 \mathrm{Y}_{61}-500 \mathrm{Y}_{71}-400 \mathrm{Y}_{81}-300 \mathrm{Y}_{91}-700 \mathrm{Y}_{101}+1 \mathrm{Q}_{11}=-300$

$-1 \mathrm{I}_{12}-400 \mathrm{Y}_{12}-200 \mathrm{Y}_{22}-1134 \mathrm{Y}_{32}-800 \mathrm{Y}_{42}-616 \mathrm{Y}_{52}-100 \mathrm{Y}_{62}-500 \mathrm{Y}_{72}-400 \mathrm{Y}_{82}-300 \mathrm{Y}_{92}-700 \mathrm{Y}_{102}+1 \mathrm{Q}_{12}=0$

$-1 \mathrm{I}_{13}-400 \mathrm{Y}_{13}-200 \mathrm{Y}_{23}-1134 \mathrm{Y}_{33}-800 \mathrm{Y}_{43}-616 \mathrm{Y}_{53}-100 \mathrm{Y}_{63}-500 \mathrm{Y}_{73}-400 \mathrm{Y}_{83}-300 \mathrm{Y}_{93}-700 \mathrm{Y}_{103}+1 \mathrm{Q}_{13}=0$

$-1 \mathrm{I}_{21}-600 \mathrm{Y}_{11}-300 \mathrm{Y}_{21}-1134 \mathrm{Y}_{31}-1200 \mathrm{Y}_{41}-616 \mathrm{Y}_{51}-200 \mathrm{Y}_{61}-750 \mathrm{Y}_{71}-400 \mathrm{Y}_{81}-600 \mathrm{Y}_{91}-1050 \mathrm{Y}_{101}+1 \mathrm{Q}_{21}=-500$

$-1 \mathrm{I}_{22}-600 \mathrm{Y}_{12}-300 \mathrm{Y}_{22}^{21}-1134 \mathrm{Y}_{32}-1200 \mathrm{Y}_{42}-616 \mathrm{Y}_{52}-200 \mathrm{Y}_{62}-750 \mathrm{Y}_{72}-400 \mathrm{Y}_{82}^{81}-600 \mathrm{Y}_{92}-1050 \mathrm{Y}_{102}+1 \mathrm{Q}_{22}=0$

$-1 \mathrm{I}_{23}+-600 \mathrm{Y}_{13}+-300 \mathrm{Y}_{23}+-1134 \mathrm{Y}_{33}+-1200 \mathrm{Y}_{43}+-616 \mathrm{Y}_{53}+-200 \mathrm{Y}_{63}+-750 \mathrm{Y}_{73}+-400 \mathrm{Y}_{83}+-600 \mathrm{Y}_{93}+-1050 \mathrm{Y}_{103}+1 \mathrm{Q}_{23}=0$

$-1 \mathrm{I}_{32}-200 \mathrm{Y}_{12}-100 \mathrm{Y}_{22}-567 \mathrm{Y}_{32}-400 \mathrm{Y}_{42}-308 \mathrm{Y}_{52}-300 \mathrm{Y}_{62}-250 \mathrm{Y}_{72}-200 \mathrm{Y}_{82}-900 \mathrm{Y}_{92}-350 \mathrm{Y}_{102}+1 \mathrm{Q}_{32}=0$

$-1 \mathrm{I}_{33}-200 \mathrm{Y}_{13}-100 \mathrm{Y}_{23}-567 \mathrm{Y}_{33}-400 \mathrm{Y}_{43}-308 \mathrm{Y}_{53}-300 \mathrm{Y}_{63}-250 \mathrm{Y}_{73}-200 \mathrm{Y}_{83}-900 \mathrm{Y}_{93}-350 \mathrm{Y}_{103}+1 \mathrm{Q}_{23}=0$

$-1 \mathrm{I}_{41}-400 \mathrm{Y}_{11}-200 \mathrm{Y}_{21}-567 \mathrm{Y}_{31}-800 \mathrm{Y}_{41}-308 \mathrm{Y}_{51}-200 \mathrm{Y}_{61}-500 \mathrm{Y}_{71}-200 \mathrm{Y}_{81}-600 \mathrm{Y}_{91}-700 \mathrm{Y}_{101}+1 \mathrm{Q}_{41}=-90$

$-1 \mathrm{I}_{42}-400 \mathrm{Y}_{12}-200 \mathrm{Y}_{22}-567 \mathrm{Y}_{32}-800 \mathrm{Y}_{42}-308 \mathrm{Y}_{52}-200 \mathrm{Y}_{62}-500 \mathrm{Y}_{72}-200 \mathrm{Y}_{82}-600 \mathrm{Y}_{92}-700 \mathrm{Y}_{102}+1 \mathrm{Q}_{42}=0$

$-1 \mathrm{I}_{43}-400 \mathrm{Y}_{13}-200 \mathrm{Y}_{23}-567 \mathrm{Y}_{33}-800 \mathrm{Y}_{43}-308 \mathrm{Y}_{53}-200 \mathrm{Y}_{63}-500 \mathrm{Y}_{73}-200 \mathrm{Y}_{83}-600 \mathrm{Y}_{93}-700 \mathrm{Y}_{103}+1 \mathrm{Q}_{43}=0$

$-1 \mathrm{I}_{51}-1000 \mathrm{Y}_{11}-500 \mathrm{Y}_{21}-3402 \mathrm{Y}_{31}-2000 \mathrm{Y}_{41}-1848 \mathrm{Y}_{51}-500 \mathrm{Y}_{61}-1250 \mathrm{Y}_{71}-1200 \mathrm{Y}_{81}-1500 \mathrm{Y}_{91}-1750 \mathrm{Y}_{101}+1 \mathrm{Q}_{51}=-250$

$-1 \mathrm{I}_{52}-1000 \mathrm{Y}_{12}-500 \mathrm{Y}_{22}-3402 \mathrm{Y}_{32}-2000 \mathrm{Y}_{42}-1848 \mathrm{Y}_{52}-500 \mathrm{Y}_{62}-1250 \mathrm{Y}_{72}-1200 \mathrm{Y}_{82}-1500 \mathrm{Y}_{92}-1750 \mathrm{Y}_{102}+1 \mathrm{Q}_{52}=0$

$-1 \mathrm{I}_{53}-1000 \mathrm{Y}_{13}-500 \mathrm{Y}_{23}-3402 \mathrm{Y}_{33}-2000 \mathrm{Y}_{43}^{2}-1848 \mathrm{Y}_{53}-500 \mathrm{Y}_{63}-1250 \mathrm{Y}_{73}-1200 \mathrm{Y}_{83}-1500 \mathrm{Y}_{93}-1750 \mathrm{Y}_{103}+1 \mathrm{Q}_{53}=0$

$-1 \mathrm{I}_{61}-4200 \mathrm{Y}_{11}-2100 \mathrm{Y}_{21}-3969 \mathrm{Y}_{31}-8400 \mathrm{Y}_{41}-2156 \mathrm{Y}_{51}-700 \mathrm{Y}_{61}-5250 \mathrm{Y}_{71}-1400 \mathrm{Y}_{81}-2100 \mathrm{Y}_{91}-7350 \mathrm{Y}_{101}+1 \mathrm{Q}_{61}=-100$

$-1 \mathrm{I}_{62}-4200 \mathrm{Y}_{12}-2100 \mathrm{Y}_{22}-3969 \mathrm{Y}_{32}-8400 \mathrm{Y}_{42}-2156 \mathrm{Y}_{52}-700 \mathrm{Y}_{62}-5250 \mathrm{Y}_{72}-1400 \mathrm{Y}_{82}-2100 \mathrm{Y}_{92}-7350 \mathrm{Y}_{102}+1 \mathrm{Q}_{62}=0$

$-1 \mathrm{I}_{63}-4200 \mathrm{Y}_{13}-2100 \mathrm{Y}_{23}^{2}-3969 \mathrm{Y}_{33}-8400 \mathrm{Y}_{43}^{42}-2156 \mathrm{Y}_{53}^{2}-700 \mathrm{Y}_{63}^{62}-5250 \mathrm{Y}_{73}-1400 \mathrm{Y}_{83}^{82}-2100 \mathrm{Y}_{93}-7350 \mathrm{Y}_{103}+1 \mathrm{Q}_{63}=0$

$-1 \mathrm{I}_{71}-1800 \mathrm{Y}_{11}-900 \mathrm{Y}_{21}-2835 \mathrm{Y}_{31}-3600 \mathrm{Y}_{41}-1540 \mathrm{Y}_{51}-1100 \mathrm{Y}_{61}-2250 \mathrm{Y}_{71}-1000 \mathrm{Y}_{81}-3300 \mathrm{Y}_{91}-3150 \mathrm{Y}_{101}+1 \mathrm{Q}_{71}=-75$

$-1 \mathrm{I}_{72}-1800 \mathrm{Y}_{12}-900 \mathrm{Y}_{22}-2835 \mathrm{Y}_{32}-3600 \mathrm{Y}_{42}^{41}-1540 \mathrm{Y}_{52}-1100 \mathrm{Y}_{62}-2250 \mathrm{Y}_{72}-1000 \mathrm{Y}_{82}-3300 \mathrm{Y}_{92}-3150 \mathrm{Y}_{102}+1 \mathrm{Q}_{72}=0$

$-1 \mathrm{I}_{73}-1800 \mathrm{Y}_{13}-900 \mathrm{Y}_{23}-2835 \mathrm{Y}_{33}-3600 \mathrm{Y}_{43}-1540 \mathrm{Y}_{53}-1100 \mathrm{Y}_{63}-2250 \mathrm{Y}_{73}-1000 \mathrm{Y}_{83}-3300 \mathrm{Y}_{93}-3150 \mathrm{Y}_{103}+1 \mathrm{Q}_{73}=0$

$-1 \mathrm{I}_{81}-1400 \mathrm{Y}_{11}-700 \mathrm{Y}_{21}-5670 \mathrm{Y}_{31}-2800 \mathrm{Y}_{41}-3080 \mathrm{Y}_{51}-1800 \mathrm{Y}_{61}-1750 \mathrm{Y}_{71}-2000 \mathrm{Y}_{81}-5400 \mathrm{Y}_{91}-2450 \mathrm{Y}_{101}+1 \mathrm{Q}_{81}=-200$

$-1 \mathrm{I}_{82}-1400 \mathrm{Y}_{12}-700 \mathrm{Y}_{22}-5670 \mathrm{Y}_{32}-2800 \mathrm{Y}_{42}-3080 \mathrm{Y}_{52}-1800 \mathrm{Y}_{62}-1750 \mathrm{Y}_{72}-2000 \mathrm{Y}_{82}-5400 \mathrm{Y}_{92}-2450 \mathrm{Y}_{102}+1 \mathrm{Q}_{82}=0$

$-1 \mathrm{I}_{83}-1400 \mathrm{Y}_{13}-700 \mathrm{Y}_{23}-5670 \mathrm{Y}_{33}-2800 \mathrm{Y}_{43}-3080 \mathrm{Y}_{53}-1800 \mathrm{Y}_{63}-1750 \mathrm{Y}_{73}-2000 \mathrm{Y}_{83}-5400 \mathrm{Y}_{93}-2450 \mathrm{Y}_{103}+1 \mathrm{Q}_{83}=0$

$100 \mathrm{Y}_{11}+50 \mathrm{Y}_{21}+340.2 \mathrm{Y}_{31}+200 \mathrm{Y}_{41}+184.8 \mathrm{Y}_{51}+50 \mathrm{Y}_{61}+125 \mathrm{Y}_{71}+120 \mathrm{Y}_{81}+150 \mathrm{Y}_{91}^{83}+175 \mathrm{Y}_{101} \Leftarrow 560$

$100 \mathrm{Y}_{12}+50 \mathrm{Y}_{22}+340.2 \mathrm{Y}_{32}+200 \mathrm{Y}_{42}+184.8 \mathrm{Y}_{52}+50 \mathrm{Y}_{62}+125 \mathrm{Y}_{72}+120 \mathrm{Y}_{82}+150 \mathrm{Y}_{92}+175 \mathrm{Y}_{102} \Leftarrow 560$

$100 \mathrm{Y}_{13}+50 \mathrm{Y}_{23}+340.2 \mathrm{Y}_{33}+200 \mathrm{Y}_{43}+184.8 \mathrm{Y}_{53}+50 \mathrm{Y}_{63}+125 \mathrm{Y}_{73}+120 \mathrm{Y}_{83}+150 \mathrm{Y}_{93}+175 \mathrm{Y}_{103} \Leftarrow 560$

$1 \mathrm{Y}_{11}+1 \mathrm{Y}_{12}+1 \mathrm{Y}_{13}=1$

$1 \mathrm{Y}_{21}+1 \mathrm{Y}_{22}+1 \mathrm{Y}_{23}=1$

$1 \mathrm{Y}_{21}+1 \mathrm{Y}_{22}+1 \mathrm{Y}_{33}=1$

$1 \mathrm{Y}_{41}^{21}+1 \mathrm{Y}_{42}^{22}+1 \mathrm{Y}_{43}^{33}=1$

$1 \mathrm{Y}_{51}+1 \mathrm{Y}_{52}+1 \mathrm{Y}_{53}=1$

$1 \mathrm{Y}_{61}+1 \mathrm{Y}_{62}+1 \mathrm{Y}_{63}=1$

$1 \mathrm{Y}_{71}+1 \mathrm{Y}_{72}+1 \mathrm{Y}_{73}=1$

$1 \mathrm{Y}_{81}+1 \mathrm{Y}_{82}+1 \mathrm{Y}_{83}=1$

$1 \mathrm{Y}_{91}+1 \mathrm{Y}_{92}+1 \mathrm{Y}_{93}=1$

$1 \mathrm{Y}_{101}+1 \mathrm{Y}_{102}+1 \mathrm{Y}_{103}=1$

$$
\mathrm{I}_{\mathrm{ij}} \geq 0 ; \mathrm{Q}_{\mathrm{ij}} \geq 0 ; \mathrm{Y}_{\mathrm{ij}}=0,1
$$

Figura 7. Modelo de programación lineal del programa de producción en ambiente de ensamble

Para las 650 unidades que se deben producir en el periodo 2 del producto $\mathrm{PI}$ se emitieron otras dos órdenes, la \#4 por 400 unidades y la \#7 por 250 unidades, ambas con fecha de entrega en el periodo

2. De manera similar, se emitieron las restantes seis órdenes de producción.

El cuadro 6 muestra la cantidad de componentes necesarios para producir cada orden emitida de acuerdo con el programa de producción. La cantidad de componentes está en función de la estructura de componentes mostrada en las figuras 2, 3 y 4.
El cuadro 7 muestra los costos de penalización asociados por entregas tardías o adelantadas para cada una de las órdenes emitidas. El programa de producción requiere que se disponga de la cantidad total de componentes de cada tipo que garantice la producción de los productos justo a tiempo para entrega.

El cuadro 7 indica que, por ejemplo, la orden \#6 fue emitida para producir 100 unidades del producto P3, el cual debe entregarse al final del periodo 2. 
Cuadro 7. Costos asociados por pedidos pendientes u órdenes adelantadas

\begin{tabular}{|c|c|c|c|c|c|c|c|c|c|c|}
\hline \multirow{2}{*}{ Periodo } & \multicolumn{8}{|c|}{ Cuadro de penalizaciones por entrega adelantada o pedido pendiente } \\
\cline { 2 - 12 } & $\mathbf{1}$ & $\mathbf{2}$ & $\mathbf{3}$ & $\mathbf{4}$ & $\mathbf{5}$ & $\mathbf{6}$ & $\mathbf{7}$ & $\mathbf{8}$ & $\mathbf{9}$ & $\mathbf{1 0}$ \\
\hline 1 & 0,0 & 0,0 & 0,0 & 3,0 & 2,0 & 1,5 & 0,9 & 2,0 & 3,5 & 2,5 \\
\hline 2 & 2,0 & 1,0 & 3,0 & 0,0 & 0,0 & 0,0 & 0,0 & 4,0 & 7,0 & 5,0 \\
\hline 3 & 4,0 & 2,0 & 6,0 & 4,0 & 3,0 & 2,0 & 1,2 & 0,0 & 0,0 & 0,0 \\
\hline
\end{tabular}

Cuadro 8. Viabilidad del programa de emisión de órdenes.

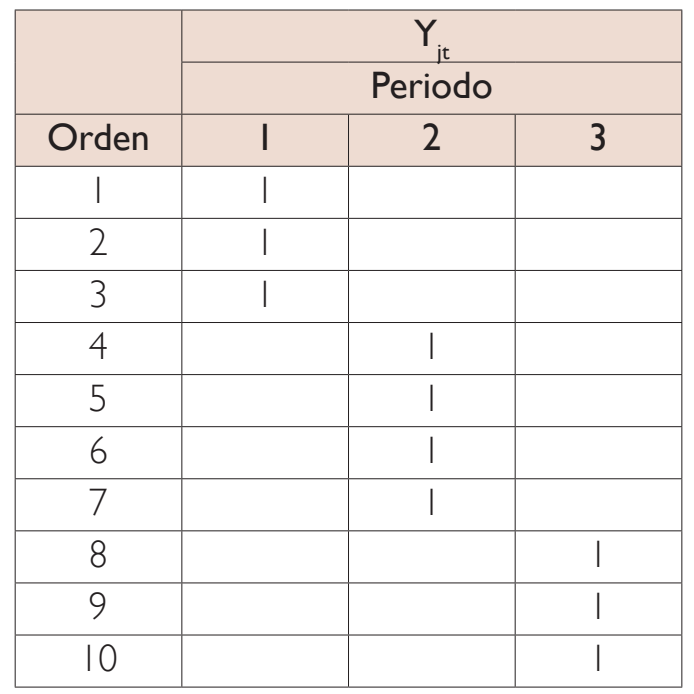

Cuadro 9. Resumen de resultados obtenidos al resolver el programa de producción de la figura 7.

\begin{tabular}{|c|c|c|c|}
\hline Componente & Periodo I & Periodo 2 & Periodo 3 \\
\hline 1 & 1434 & 2016 & 1400 \\
\hline 2 & 1534 & 2766 & 2050 \\
\hline 3 & 717 & 1258 & 1450 \\
\hline 4 & 1077 & 1808 & 1500 \\
\hline 5 & 4652 & 5598 & 4450 \\
\hline 6 & 10169 & 16506 & 10850 \\
\hline 7 & 5460 & 8490 & 7450 \\
\hline 8 & 7570 & 9430 & 9850 \\
\hline
\end{tabular}

Se penaliza con 1.50 unidades monetarias si se entrega en el periodo I (adelantado) o con 2.0 unidades monetarias si se entrega en el periodo 3 (atrasado).

El modelo de programación lineal (Baker, 1990) que se muestra en la figura 7 determina si el programa de emisión de órdenes de el cuadro 5, el cual se propuso con base en el plan de producción mostrado en el cuadro 4, es viable. Si el programa no es viable, el modelo de programación lineal propone un programa viable y determina la cantidad de componentes totales necesarios en cada periodo para cumplir con el programa de producción, minimizando los costos totales de mantenimiento del inventario de componentes, así como los costos de penalización de las órdenes por entregas tempranas o tardías. Para la función objetivo, el modelo se alimenta de la estructura de costos presentada en el cuadro 7. 
Para la construcción de las restricciones se utilizó la estructura de componentes presentada en el 24 restricciones determinan el inventario final de los ocho tipos de componentes, las siguientes tres restricciones garantizan que la producción no exceda la cantidad de horas disponibles por mes y el restante grupo de restricciones indican que cada una de las órdenes emitidas se produce completa en un solo periodo.

Los cuadros 8 y 9 resumen los resultados obtenidos al resolver el problema en el programa de computadora OR Brainware Decision Tools. El cuadro 8 indica que el programa de emisión de órdenes establecido en el cuadro 5 es viable, pues las órdenes de producción se terminan en las fechas de entrega pactadas.

El cuadro 9 muestra los componentes totales que deben estar disponibles en cada uno de los periodos del horizonte de programación para producir todos los productos. Por ejemplo, en el periodo I se deben tener disponibles 4652 componentes \#5. Esta cantidad de componentes se desglosa de la siguiente manera: el programa de producción indicó que en el periodo I se deben producir 200 y 100 unidades del producto $\mathrm{PI}$, emitidas en las órdenes de producción \# I y \#2.

La estructura del producto $\mathrm{PI}$ indica que se requieren dos componentes SI por cada unidad de producto $\mathrm{PI}$. A su vez, se requieren dos componentes \#5 por cada componente $\mathrm{SI}$. Además se requiere para este producto $\mathrm{PI}$ un componente $\mathrm{S} 3$, el cual a su vez requiere un componente $\# 5$, obteniéndose para este producto un total de 1500 componentes. Adicionalmente, el programa de producción establece que deben producirse 567 unidades del producto P2 en el periodo I.

Este producto también requiere de componentes \#5. La estructura del producto P2 muestra que se requieren dos componentes $\mathrm{SI}$ por cada unidad de producto $\mathrm{P} 2$, y el componente $\mathrm{S} 2$ requiere a su vez dos componentes \#5. También se requiere un componente S3 y un componente S4, los cuales a su vez requieren un componente \#5 cada uno.
Esto da un total de 3402 componentes \#5 para el producto P2, lo cual sumado a los 1500 componentes \#5 requeridos por el producto $\mathrm{PI}$ da un total de 4902 componentes \#5. Sin embargo, se tiene un inventario inicial de 250 componentes \#5, por lo cual el total requerido para este componente en el periodo \#I es de 4652 unidades.

Si se tienen pedidos comprometidos con clientes, el programa de producción (Domínguez et al., 1995) debe considerar la cantidad más grande entre el pronóstico de la demanda y estos pedidos.

Finalmente, el cuadro 8 generó la recepción de pedidos planificados de los componentes. En consecuencia, si se conocen los tiempos de suministro por parte de los proveedores para estos componentes, se puede realizar la emisión de los pedidos planificados.

\section{Conclusión}

Se concluye que el modelado mediante la programación lineal es una poderosa herramienta para realizar el planeamiento y la programación de la producción en ambientes de ensamble complejos, con el objetivo de apoyar el proceso de toma de decisiones. Esto permite a las empresas obtener ventajas competitivas en términos de reducción de costos, cumplimiento de las fechas de entrega y planificación de los inventarios. Además, el proceso de toma de decisiones se fortalece con la aparición de nuevas herramientas computacionales capaces de resolver modelos de gran escala a un bajo costo.

\section{Bibliografía}

Domínguez, J. A.; García, S; Ruiz, A; Domínguez, M.A \& Álvarez, M. J. (1995). Dirección de Operaciones. Madrid: McGraw Hill.

Moya, M.J. (201 I). Planeamiento de la producción con incertidumbre mediante Programación Lineal: Uso de OR Brainware Decision Tools. Tecnología en Marcha, Vol 10. Num. 2 Cartago: Editorial Tecnológica de Costa Rica.

Sipper, D., \& Bulfin Jr. R. (1998). Planeación y Control de la Producción. Vol. I. México: Mc Graw Hill. 